Research Paper

\title{
MiR-539-5p negatively regulates migration of rMSCs induced by Bushen Huoxue decoction through targeting Wnt5a
}

Liuchao $\mathrm{Hu}^{1}$, Yamei Liu ${ }^{2,3}$, Bin Wang ${ }^{1}$, Zhifang $\mathrm{Wu}^{1}$, Yingxiong Chen ${ }^{1}$, Lijuan $\mathrm{Yu}^{2,3}$, Junlang Zhu1, Wei Shen ${ }^{1}$, Chen Chen 2,3 , Dongfeng Chen ${ }^{2,3}$, Gang Li ${ }^{4}$, Liangliang $\mathrm{Xu}^{5,6}{ }^{\bowtie}$, Yiwen Luo ${ }^{\circledR}$

1. Department of Traumatology, The Third Affiliated Hospital of Guangzhou University of Chinese Medicine, Guangzhou, Guangdong 510240, P.R. China.

2. School of Basic Medical Science, Guangzhou University of Chinese Medicine, Guangzhou 510006, P.R. China.

3. The Research Center of Basic Integrative Medicine, Guangzhou University of Chinese Medicine, Guangzhou 510006, P.R. China.

4. Department of Orthopaedics \& Traumatology, Faculty of Medicine, The Chinese University of Hong Kong, Prince of Wales Hospital, Shatin, Hong Kong, P.R. China

5. Key Laboratory of Orthopaedics \& Traumatology, The First Affiliated Hospital of Guangzhou University of Chinese Medicine, Guangzhou University of Chinese Medicine, Guangzhou, P.R. China.

6. Laboratory of Orthopaedics \& Traumatology, Lingnan Medical Research Center, Guangzhou University of Chinese Medicine, Guangzhou, P.R. China

$\triangle$ Corresponding authors: The Third Affiliated Hospital of Guangzhou University of Chinese Medicine, Guangzhou University of Chinese Medicine, Guangzhou, China (YW. Luo); The First Affiliated Hospital of Guangzhou University of Chinese Medicine, Guangzhou University of Chinese Medicine, Guangzhou, China ( LL. Xu). E-mail addresses: gzzyydxlyw@126.com (YW. Luo), xull-2016@gzucm.edu.cn (LL. Xu).

(c) Ivyspring International Publisher. This is an open access article distributed under the terms of the Creative Commons Attribution (CC BY-NC) license (https://creativecommons.org/licenses/by-nc/4.0/). See http://ivyspring.com/terms for full terms and conditions.

Received: 2019.01.23; Accepted: 2019.04.24; Published: 2019.06.10

\begin{abstract}
Bone fractures are very common, and above $5 \%$ of the fractures are impaired, leading to nonunions and severe disablilities. The traditional Chinese medicine Bushen Huoxue decoction (BHD) has been used to treat fracture in China. Our previous report has found that BHD promotes migration of rat mesenchymal stem cells (rMSCs) by activating Wnt5a signaling pathway. However, whether and how miRNAs are involved in modulating rMSCs migration induced by BHD has not been explored. In the present study, miRNA microarray analysis and further validation by real-time quantitative RT-PCR revealed that miR-539-5p was down-regulated in BHD-induced $r M S C s$. Transfection of miR-539-5p mimics suppressed rMSCs migration while the miR-539-5p inhibitor promoted rMSCs migration. Our results suggested that miR-539-5p was a negative regulator of migration of rMSCs induced by BHD. Target prediction analysis tools and Dual-luciferase reporter gene assay identified $\mathrm{Wnt5a}$ as a direct target of miR-539-5p. MiR-539-5p inhibited the expression of the Wnt5a and its downstream signaling molecules including JNK, PKC and CaMKII, which played a critical role in regulating migration of rMSCs. Taken together, our results demonstrate that miR-539-5p negatively regulates migration of $r M S C$ s induced by BHD through targeting Wnt5a. These findings provide evidence that miR-539-5p should be considered as an important candidate target for the development of preventive or therapeutic approaches against bone nonunions.
\end{abstract}

Key words: miR-539-5p, Wnt5a, rat mesenchymal stem cells (rMSCs), migration

\section{Introduction}

Fracture nonunion is a devastating complication encountered by repair of bone fracture and bone defects [1]. In the United States, approximately 7.9 million patients sustain fractures annually, and up to $10 \%$ of these patients may suffer subsequently from a delayed union or a nonunion at the fracture site [2]. Successful fracture healing is a complicated and well-orchestrated regeneration process comprising inflammatory, repair, and remodeling phases $[3,4]$.
This process is relied on a large number of Mesenchymal stem cells (MSCs), which can be induced to differentiate into osteoblasts in vitro and in vivo and form bone [5-7]. Once specific signals are released from injured tissue, MSCs are stimulated to leave their niche and migrate to the target tissues to proliferate and differentiate into mature cells. This process is defined as MSCs homing and considered a natural self-healing response $[8,9]$. MSCs is a 
promising cell source for tissue engineering, particularly for bone regeneration. Previous studies showed that both systemic and local injection of allogenic MSCs promoted fracture healing [10, 11]. MSCs have the capacity to enhance fracture healing in bone fractures when delivered to the fracture site [12].Therefore, enhancing MSCs migration capacity is essential for optimizing the therapeutic outcome.

Our recent report indicated that Bushen Huoxue decoction (BHD), a Chinese traditional medicine formula, promoted migration of rMSCs by activating Wnt5a $[13,14]$. BHD has been previously confirmed to have good efficacy in treating bone diseases such as osteoarthritis and osteoporosis [15, 16]. Three main herbs are included in BHD, namely Rehmannia glutinosa, Cuscuta chinensis and Fructus psoraleae, which play important roles in osteoblastic bone formation [17-19]. Wnt5a, one of the most extensively studied Wnt proteins of Wnt family, has been well known to regulate cell adhesion, migration, and polarity [20-22]. However, the underlying mechanism of BHD-induced rMSCs migration is still unknown.

Recently, it is increasingly recognized that miRNAs are important regulators of migration of MSCs[23]. Several studies have reported that miRNAs target the critical cell signaling pathway involved in migration of MSCs. For example, multiple miRNAs, including miR-27b, miR-27a, miR-146a-5p and miR-886-3p, have been reported to suppress the migration of MSCs through targeting SDF-1a/CXCR4 axis [24-26]. miR-221 and miR-26b promotes migration of MSCs through activation of Akt and FAK [27]. MiRNAs not only acts as a positive regulator but also negative regulators that suppress migration of MSCs. Thus, miRNAs play critical roles in migration of MSCs. miR-539-5p has been found to function as a suppressor in tumor cell migration and invasion [28, 29]. However, it has not yet been explored whether miR-539-5 $p$ regulates MSCs migration.

In the present study, we used miRNA microarray analysis and real-time quantitative RT-PCR to explore the differentially expressed miRNAs in BHD-treated rMSCs. We found that mir-539-5p was the most significantly inhibited microRNA. In addition, we revealed that miR-539-5p was a key negative regulator of migration of rMSCs through targeting Wnt5a.

\section{Materials and Methods}

\section{rMSCs isolation and culture}

This experiment was approved by the Animal Care and Use Committee of Guangzhou University of Chinese Medicine. rMSCs were isolated and cultured as described previously [13]. In briefly, the bone marrow of the bilateral femoral was flushed out and cultured in a-MEM (HyClone), 10\% FBS (Gibco), and $1 \%$ penicillin-streptomycin solution (HyClone) at $37^{\circ} \mathrm{C}$ with $5 \% \mathrm{CO}^{2}$. rMSCs from passage 3 or 5 were used for analysis.

\section{rMSCs characterization}

The cell surface markers (CD90, CD45, CD44 and CD34) were confirmed by flow cytometry. rMSCs were analyzed for osteogenic, and adipogenic differentiation in vitro to determine multipotency according to standard conditions, as described previously [13].

\section{BHD preparation}

BHD contains Eleven Chinese herbs (Rehmannia glutinosa 18g, Cuscuta chinensis 18g, Fructus Psoraleae 18g, Eucommia ulmoides 6g, Fructus Corni 6g, Herba Cistanches 6g, Fructus Lycii 6g, Radix Angelicae Pubescentis 6g, Radix Angelicae Sinensis 6g, Myrrha 6g, and Flos Carthami 3g), which is purchased from The Third Affiliated Hospital of Guangzhou University of Chinese Medicine. BHD was extracted using the Soxhlet extraction method in petroleum, as described previously [13].

\section{Treatment of rMSCs with BHD}

rMSCs were seeded at a density of $8 \times 10^{4}$ cells/well in 6-wellculture plates. When $80 \%$ confluence was reached, cells were treated with a-MEM containing $100 \mu \mathrm{g} / \mathrm{ml} \mathrm{BHD}$ (the optimum concentration of previous studies) for $24 \mathrm{~h}$ at $37^{\circ} \mathrm{C}$ in $5 \% \mathrm{CO}^{2}$. rMSCs cultured with a-MEM only were used as a control. After $24 \mathrm{~h}$, the cells were extracted for RNA extraction.

\section{MiRNA microarray analysis}

Total RNA was extracted using miRcute miRNA Isolation Kit (Tiangen, Beijing) according to the manufacturer's instructions. The extracted RNA was quantified by NanoDrop ND-2000 (Thermo Scientific). RNA integrity was assessed using Agilent Bioanalyzer 2100 (Agilent Technologies). The preparation of whole transcriptome libraries and deep sequencing were performed by the Annoroad Gene Technology Corporation (Beijing, China).

\section{Real-time quantitative RT-PCR}

The miRNAs were enriched using miRcute miRNA Isolation Kit (Tiangen, Beijing) according to the manufacturer's instructions. The concentration and purity of RNA were measured using Nanodrop 2000. cDNA was synthesized with miRcute Plus miRNA First-Strand cDNA Kit. Real-time quantitative RT-PCR was performed using miRcute Plus miRNA 
qPCR Kit (Tiangen, Beijing) .U6 RNA was used as an internal parameter to determine the relative expression. Total RNA of rMSCs was also extracted using TRIzol Reagent (Invitrogen). cDNA was synthesized with PrimeScript RT Master Mix (TaKaRa). Expression of Wnt5a was measured by qRT-PCR. Real-time quantitative RT-PCR was performed using the SYBR Premix Ex Taq II (TaKaRa). GAPDH was used as as an endogenous control. Primer sequences were shown in Table 1.

Table 1. List of primer sequences for real-time quantitative RT-PCR

\begin{tabular}{ll}
\hline Primer name & Sequence $\left(5^{\prime}-3^{\prime}\right)$ \\
\hline miR-376b-5p & GTGGATATTCCTCTATGGTTA \\
let-7b-3p & CTATACAACCTACTGCCTTCCC \\
miR-409a-5p & AGGTTACCCGAGCAACTTTGCAT \\
miR-3102 & CTCTACTCCCTGCCCAGCCA \\
miR-539-5p & GGAGAAATTATCCTTGGTGTGT \\
miR-1843a-3p & TCTGATCGTTCACCTCCATACA \\
miR-137-3p & TTATTGCTTAAGAATACGCGTAG \\
miR-216b-5p & AAATCTCTGCAGGCAAATGTGA \\
miR-223-5p & CGTGTATTTGACAAGCTGAGTTG \\
miR-211-3p & GGCAAGGACAGCAAAGGGGG \\
miR-194-3p & CCAGTGGGGCTGCTGTTATCT \\
miR-147 & GTGTGCGGAAATGCTTCTGCTA \\
U6 & GCTTCGGCAGCACATATACTAAAAT \\
Wnt5a forward & CGAAGACGGGCATCAAAGA \\
Wnt5a reverse & TGCATCACCCTGCCAAAGA \\
JNK forward & GGAGCGAACTAAGAATGGCG \\
JNK reverse & CATGTCATTGACAGACGGCG \\
CaMKII forward & ATGGATGGAAATGGAATGCC \\
CaMKII reverse & CCCCGAACGATGAAAGTGAA \\
PKC forward & AAGGTGGTCCACGAGGTGAA \\
PKC reverse & TTCCAATGCCCCAGATGAAG \\
GAPDH forward & AGGGCTGCCTTCTCTTGTGA \\
GAPDH reverse & AACTTGCCGTGGGTAGAGTCA \\
\hline
\end{tabular}

\section{Western blot analysis}

rMSCs were lysed with RIPA buffer containing protease and phosphatase inhibitors (Biyotime). The protein concentration was measured by a BCA Protein Assay kit (Biyotime). Equal amounts of protein were separated by SDS-PAGE, transferred to a PVDF membrane, blocked in 5\% milk, and immunoblotted with primary antibodies overnight at $4^{\circ} \mathrm{C}$. The membranes were washed in TBST and incubated with a corresponding secondary antibody for $1 \mathrm{~h}$ at room temperature. Protein bands were visualized using an enhanced chemiluminescence kit (Pierce). The following primary antibodies were used: Wnt5a (1:300, Abcam) and $\beta$-actin (1:1,000, CST).

\section{Transfections of rMSCs with miR-539-5p mimics, inhibitor or siWnt5a}

rMSCs were plated into 6 well cell culture cluster at a density of $1.5 \times 10^{5}$ cells per well and transfected with $50 \mathrm{nM}$ miR-539-5p mimics, $100 \mathrm{nM}$ inhibitor or 80nM siWnt5a (GenePharma, Shanghai, China) using lipo2000 Transfection Agent (Invitrogen, USA) according to the manufacturer's instructions. The cells were collected after the terminal transfection for $24 \mathrm{~h}$ for analysis. Three sequences for siRNA targeting Wnt5a were shown in Table 2.

Table 2. Sequences of siRNA targeting Wnt5a.

\begin{tabular}{lll}
\hline Gene & Sense Primer Sequence $\left(5^{\prime}-3^{\prime}\right)$ & Antisense Primer Sequence $\left(5^{\prime}-3^{\prime}\right)$ \\
\hline siWnt5a & GGUCCCUAGGUAUGAAUAATT & UUAUUCAUACCUAGGGACCTT \\
-1 & & \\
siWnt5a & GCAGCCGAGAGACAGCCUUTT & AAGGCUGUCUCUCGGCUGCTT \\
-2 & & \\
siWnt5a & CCACGCCAAGGGCUCCUAUTT & AUAGGAGCCCUUGGCGUGGTT \\
-3 & &
\end{tabular}

\section{Cell migration assay}

Cell migration ability was evaluated by transwell assay and wound healing assay. For transwell assay, cells were pretreated by different condition including BHD, or transfection of miR-539-5p mimics, inhibitors or siWnt5a. Then, cells $\left(8 \times 10^{4}\right.$ cells/well) were plated to upper chamber of Transwell plates (Corning Costar) in a serum-free medium with $10 \%$ FBS containing the medium at the bottom layer. After incubating for $10 \mathrm{~h}$ at $37{ }^{\circ} \mathrm{C}$, rMSCs at the upper layer of the membrane were scraped and rMSCs at the lower layer were stained with $0.5 \%$ Crystal Violet Staining Solution and photographed under a microscope. A number of cells were quantified in the randomly selected fields. For wound healing assay, rMSCs were incubated in $6 \mathrm{~cm}$ dish and cultured until 95\% confluence. A scratch wound was created with a micropipette tip. The cells were photographed and counted under a phase contrast microscope.

\section{Bioinformatic Analysis}

TargetScan (http://www.targetscan.org) and MiRanda (http://www.microrna.org) were used in the bioinformatic analysis of miRNAs. The target genes were verified using in vitro experiments.

\section{Luciferase reporter assay}

Luciferase Reporter Assay was performed using Dual-Luciferase Reporter Assay System (Promega, Madison, WI, USA) according to the manufacturer's instructions. In briefly, wild-type and mutant Wnt5a (without miR-539-5p binding sites) plasmids pmiR-RB-Report ${ }^{\mathrm{TM}}$ were co-transfected with miR-539-5p mimics or mimics NC into 293T cells using Lipofectamine 2000 (Invitrogen). The luciferase activity was measured at $48 \mathrm{~h}$ after transfection using GloMax ${ }^{\mathrm{TM}}$ 20/20 Single tube luminometer (Promega, Madison, WI, USA).

\section{Statistical analysis}

Data is presented for each group as means \pm standard deviation (SD). Analysis was performed 
using SPSS16.0 software. Differences between groups were compared by t-tests or one-way analysis of variance (ANOVA). $P<0.05$ was considered to be statistically significant.

\section{Results}

\section{Characterization of rMSCs}

The cultured cells were spindle-shaped and exhibited the typical morphology of stem cells (Supplementary Fig. 1A). Alizarin red staining showed that intracellular calcium nodule formation (Supplementary Fig. 1B). Oil red staining showed the formation of red small droplets of oil in cell (Supplementary Fig. 1C). Flow cytometry showed that Cells positive expression of CD90 (98.58\%), CD44 $(95.50 \%)$ and negative for CD45 $(0.24 \%)$, CD34 $(0.31 \%)$ (Supplementary Fig. 1D).

\section{Identification of BHD-responsive miRNAs in rMSCs}

The present study aimed to determine if differential miRNA expression existed in rMSCs following treatment with BHD. miRNA microarray analysis is an effective method for the prediction of the mechanisms underlying the effects of Chinese medicine. The up- and down-regulated miRNA (fold change $\geq 2.0$ ) from miRNA microarray have been uploaded in the supplementary data (Supplementary Table 1). A total of 70 differentially expressed miRNAs were identified between the control and BHD groups. Compared with the control, 27 miRNAs were upregulated and 43 miRNAs were downregulated in the BHD groups. The Heatmap were shown in (Fig. 1A). We chose some significantly different miRNAs for further verification. The most interesting one was miR-539-5p as its expression was significantly downregulated in BHD groups (Fig. 1B). The function of miR-539-5p in migration of rMSCs is largely unknown, so further in vitro analysis of miR-539-5p was conducted.

\section{MiR-539-5p negatively regulates migration of rMSCs}

To evaluate the role of miR-539-5p in migration of rMSCs, we transfected rMSCs with mimics NC, miR-539-5 $p$ mimics, inhibitor NC or miR-539-5 $p$ inhibitor respectively. The result revealed that the migration ability of rMSCs significantly decreased in the miR-539-5p mimics group, compared with the mimics NC group and increased in the miR-539-5p inhibitor group compared with the inhibitor NC group. The results of transwell assay and wound healing assay shown in (Fig. 2A-D). Real-time quantitative RT-PCR showed that miR-539-5p expression decreased in the miR-539-5p mimics group, compared with the mimics NC group and increased in the miR-539-5p inhibitor group compared with the inhibitor NC group (Fig. 2E).

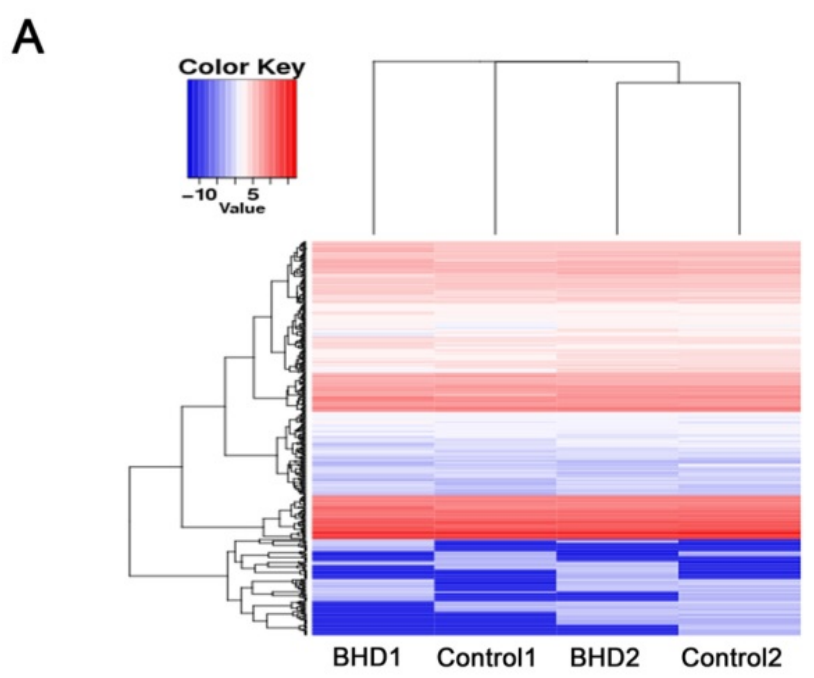

\section{B}

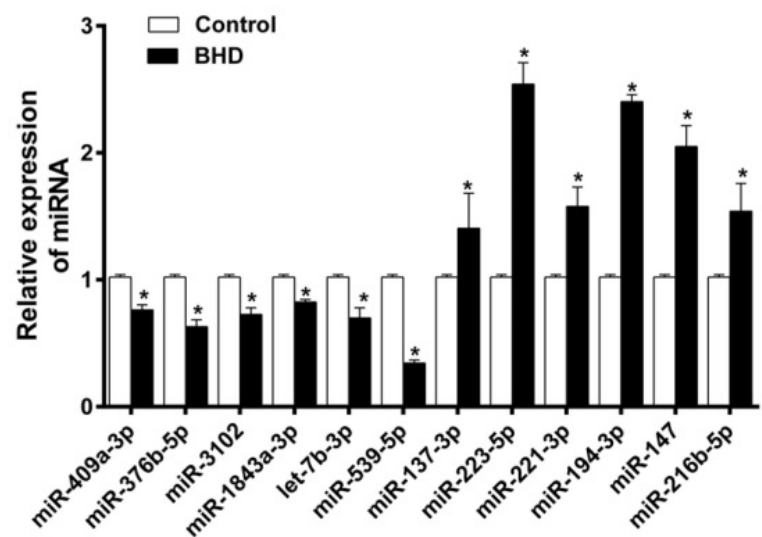

Figure 1. The differentially expressed miRNAs in rMSCs treated with BHD. (A) Heatmap depicting expression levels of miRNAs between control and BHD-treated rMSCs. Compared with control group, there were 27 miRNA up-regulated and 43 down-regulated in BHD induction group (fold change $\geq 2.0$ ). (B) Among the miRNAs found by microarray analysis, we made further screening and selection by real-time quantitative RT-PCR. Data are presented as mean \pm SD $(n=3$, $\left.{ }^{*} P<0.05\right)$

\section{Wnt5a is a potential target of miR-539-5p}

To gain insight into the molecular mechanisms by which miR-539-5p regulates the migration of rMSCs, we predicted the potential targets of miR-539-5p using Miranda and TargetScan. We found that migration-related gene-Wnt5a had a miR-539-5p binding site in its $3^{\prime} \mathrm{UTR}$ region (Fig. 3A-B). To test whether miR-539-5p directly targets this gene, we constructed luciferase reporters that had either a wild-type (WT) 3'UTR or a 3'UTR containing mutant (MUT) sequences of the miR-539-5p binding site. 293T Cells were co-transfected with the luciferase reporter carrying WT Wnt5a 3'UTR and MUT Wnt5a 3'UTR plasmids, as well as the miR-539-5p mimics, or mimics NC. We found that miR-539-5p mimics 
A

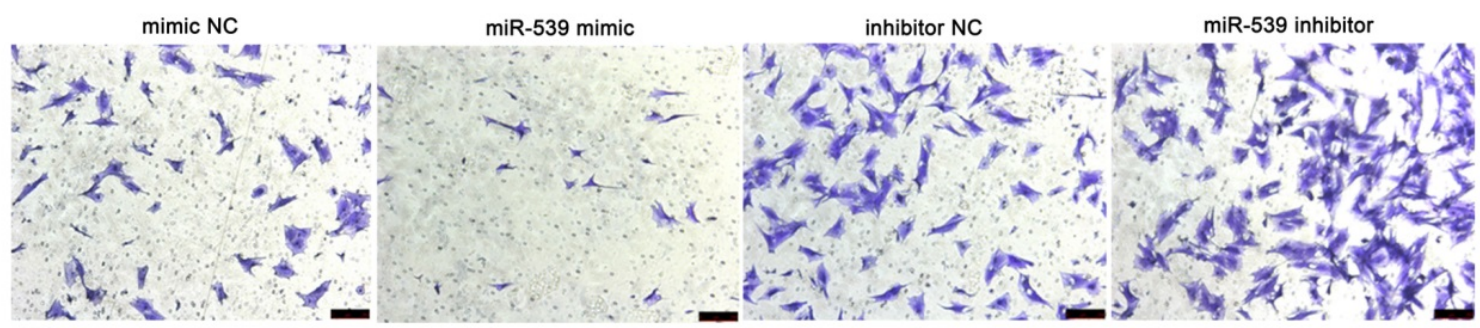

B

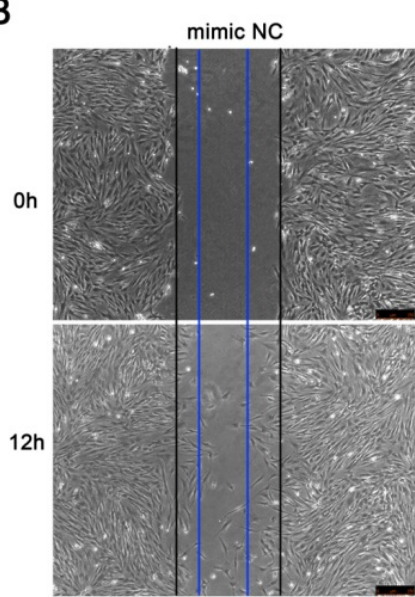

C

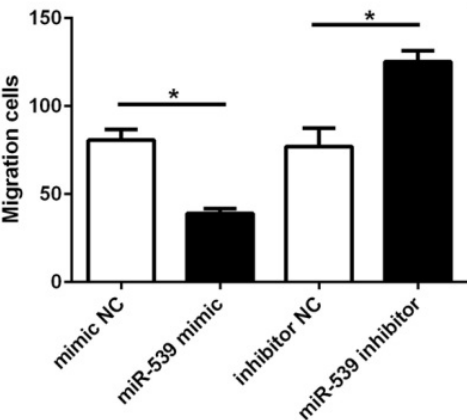

miR-539 mimic

inhibitor NC

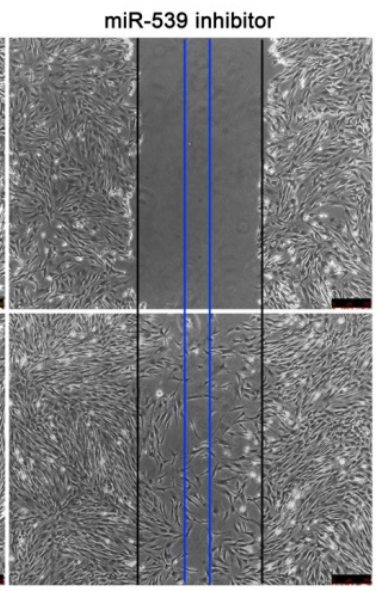

D

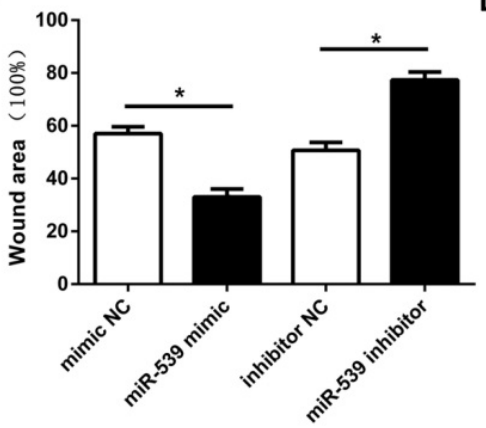

E

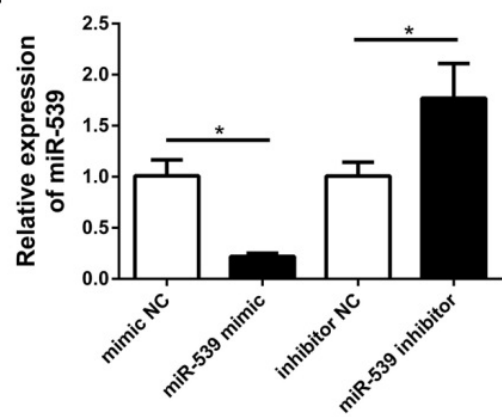

Figure 2. MiR-539-5p negatively regulated migration of rMSCs. The mimics NC, miR-539 mimics, inhibitor NC or miR-539 inhibitor was transfected into rMSCs for 24h. (A) Transwell assay.The rMSCs were seeded in the upper layer of migratory chamber to perform the transwell assay after transfection. Crystal violet staining was used to detect the rMSCs migrated through the membrane. (B) Wound healing assay. An artificial homogenous wound was made after transfection. Black and blue lines indicated start and end $(12 \mathrm{~h}$ ) positions of $\mathrm{rMSCs}$ after scraping. (C) The number of migrated cells were quantified by averaging five random fields per well under microscope ( $\mathrm{n}=5$, * $P<0.05$ ). (D) Quantitative results of wound healing assays. The scratch area was observed under a phase contrast microscope and photographed ( $n=3$, $\left.{ }^{*} P<0.05\right)$. (E) Real-time quantitative RT-PCR detection of miR-539 expression in mimics NC, miR-539 mimics, inhibitor NC or miR-539 inhibitor transfected $r M S C s(n=3$, *P<0.05). All data are presented as mean \pm SD.

remarkably inhibited the luciferase reporter activity of the WT Wnt5s 3'UTR, but not that of the MUT 3'UTR (Fig. 3C). Western blot and real-time quantitative RT-PCR results revealed that Wnt5a expression at protein (Fig. 3D-E) and mRNA levels (Fig. 3F) was found to be markedly decreased in the presence of miR-539-5p mimics group compared with mimics NC group. These results suggested that miR-539-5p directly regulates the expression of Wnt5a.

\section{Silence of Wnt5a inhibited rMSCs migration}

We transfected siRNA to knock-down Wnt5a to determine whether the Wnt5a contributes to the migration of rMSCs. Three siRNAs were transfected into rMSCs and found that siWnt5a-1 significantly decreased the expression of Wnt5a mRNA (Fig. 4A).
So we chose siWnt5a-1 for further experiment. As expected, knock-down of Wnt5a resulted in a significantly decreased in cell migration (Fig. 4B-C). Consistently, the expression of Wnt5a protein was significantly downregulated after transferring siWnt5a -1 (Fig. 4D-E).

\section{MiR-539-5p regulated BHD-induced migration of rMSCs}

To further investigate the functional roles of miR-539-5 $p$ in the directed migration of rMSCs, we then transfected rMSCs with miR-539-5p mimics, or inhibitor in the presence or absence of BHD. In preliminary experiments, we determined that the optimum concentration for promoting cell migration is $100 \mu \mathrm{g} / \mathrm{ml}$ [13]. Therefore, we chose the 
concentration of $100 \mu \mathrm{g} / \mathrm{ml}$ to assess cell migration. As shown in (Fig. 5A-B), we transfected rMSCs with miR-539-5p mimics, which led to rMSCs migration decreased and BHD-induced migration was also dramatically reduced. We then transfected rMSCs with miR-539-5p inhibitor and resulted in an increased migration in BHD-induced group while

A

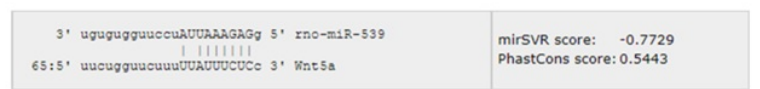

B

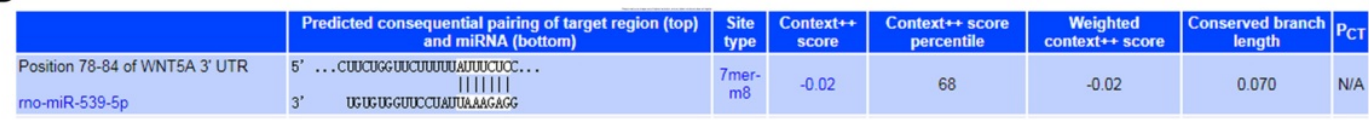

D

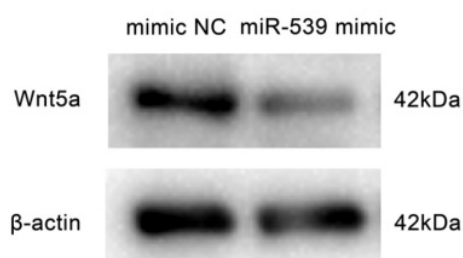

E

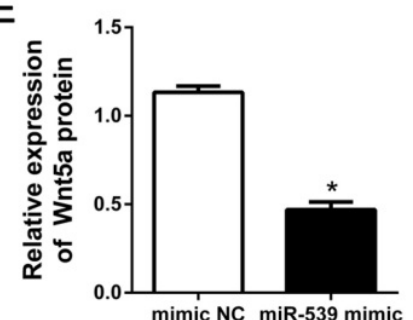

cells migrated through the filter at a low rate in the absence of BHD. Quantitative analysis confirmed that rMSCs with lower level of miR-539-5p showed much stronger migration capacity (Fig. 5C-D). These results suggested that miR-539-5p regulated BHD-induced migration of rMSCs.

C

R-Wnt5a-WT 5'cugguucuuuuuAUUUCUCc.. R-Wnt5a-MUT 5'cugguucuuuuuUAAAGAGc...

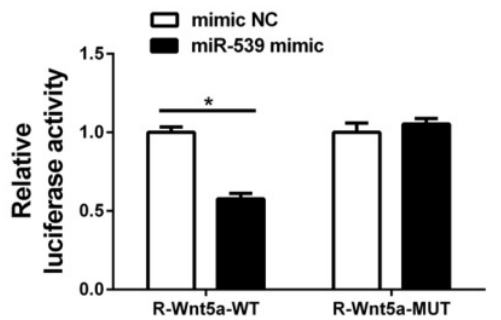

$\mathbf{F}$

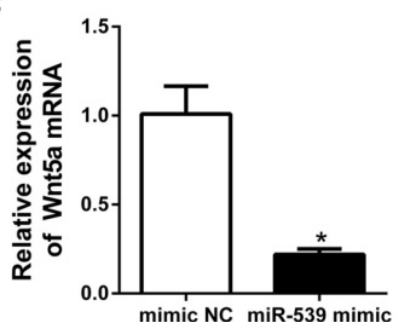

Figure 3. Wnt5a was a target gene of miR-539-5p. (A) MiRanda prediction showed that miR-539-5p bound to 3'UTR of Wnt5a. (B) TargetScan prediction showed that miR-539-5p bound to 3'UTR of Wnt5a. (C) The luciferase activity was decreased after treatment by a combination of miR-539-5p mimics and Wnt5a-3'UTR-WT, suggesting that miR-539-5p regulated Wnt5a $(n=3, * P<0.05)$. (D) Western blot detection of Wnt5a protein expression in mimics NC or miR-539-5p mimics transfected $r$ MSCs. (E) Quantification of the bands intensity using Image J software. The protein level was normalized to $\beta$-actin $\left(n=3\right.$, $\left.{ }^{*} P<0.05\right)$. (F) Real-time quantitative RT-PCR detection of Wnt5a mRNA expression in mimics NC or miR-539-5p mimics transfected rMSCs $\left(n=3,{ }^{*} P<0.05\right)$. All data are presented as mean \pm SD.

A

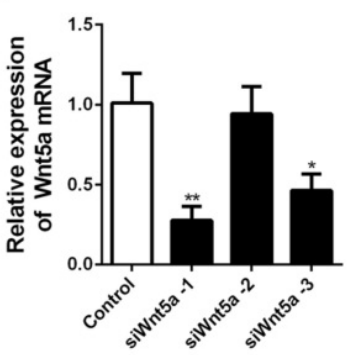

C

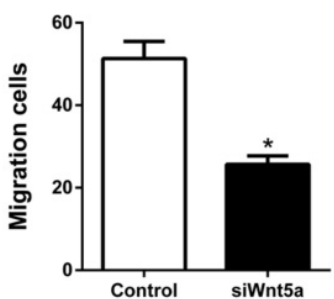

B

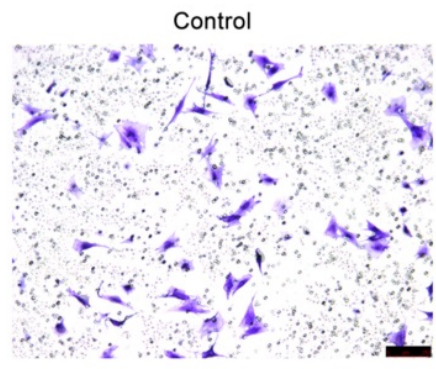

D

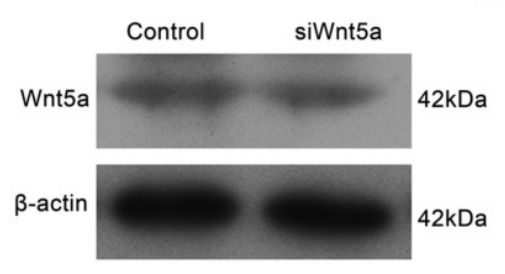

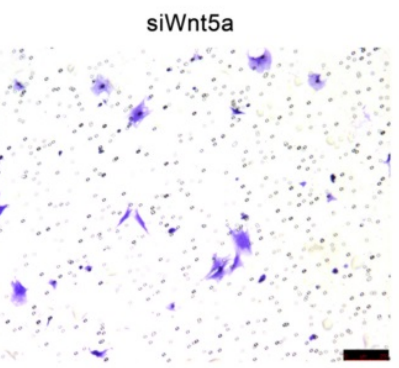

$\mathrm{E}$

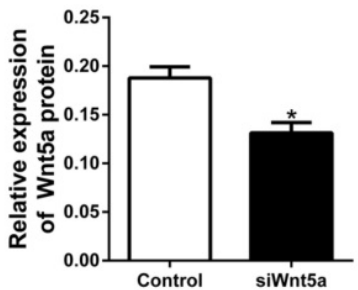

Figure 4. Silence of Wnt5a inhibited rMSCs migration. (A) The siRNA targeting Wnt5a was transfected into rMSCs as mentioned in Materials and Methods. siWnt5a-1 showed the best knockdown efficiency $\left(n=3,{ }^{*} P<0.05,{ }^{* * *} P<0.01\right)$. (B) Transwell assay. $r M S C s$ were transfected with siWnt5a or negative control and were stained with] crystal violet. (C) The number of migrated cells were quantified by averaging five random fields per well under microscope $\left(n=5\right.$, $\left.{ }^{*} P<0.05\right)$. (D) $r M S C s$ were transfected with siWnt5a or negative control. Total proteins were extracted from rMSCs and analyzed by western blot using indicated antibodies. $\beta$-actin was used as loading control. The experiments were repeated three times. (E) Quantification of the bands intensity using Image J software $\left(n=3,{ }^{*} P<0.05\right)$. All data are presented as mean \pm SD. 
A

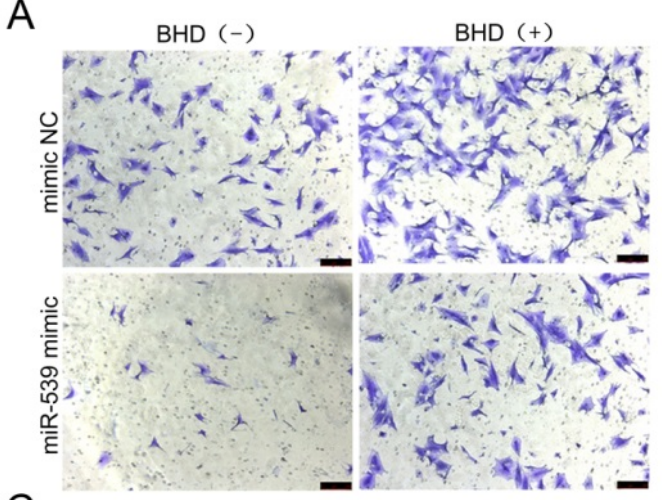

C

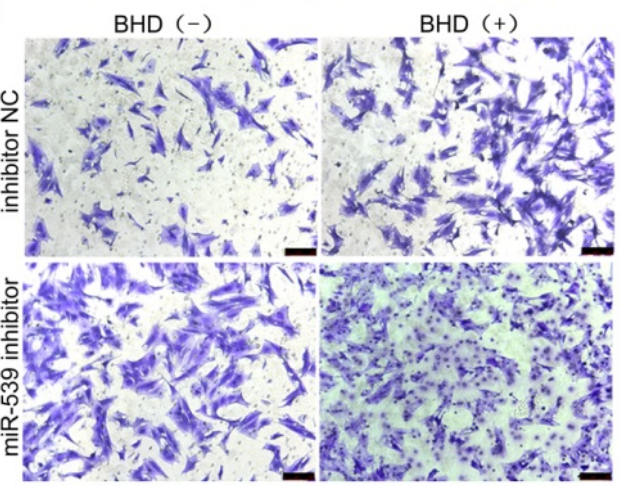

B

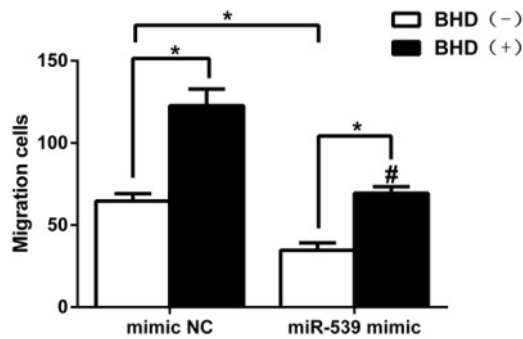

D

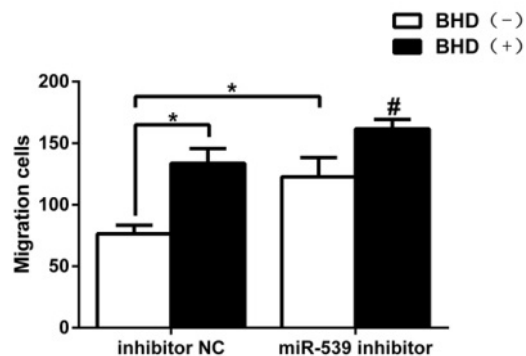

Figure 5. MiR-539-5p regulated rMSCs migration induced by BHD. (A) Transwell assay. $r$ MSCs infected with mimics NC or miR-539 mimics in the presence or absence of $100 \mu \mathrm{g} / \mathrm{ml} \mathrm{BHD}$ and were stained with crystal violet. (B) The number of migrated cells were quantified by averaging five random fields per well under microscope ( $\mathrm{n}=5$, $\# P<0.05$ vs rMSCs transfected with mimics NC with BHD induction, $\left.{ }^{*} P<0.05\right)$. (C) Transwell assay. rMSCs infected with inhibitor NC or miR-539 inhibitor in the presence or absence of $100 \mu \mathrm{g} / \mathrm{ml} \mathrm{BHD}$ and were stained with crystal violet. (D) The number of migrated cells were quantified by averaging five random fields per well under microscope ( $\mathrm{n}=5$, $\# P<0.05$ vs rMSCs transfected with inhibitor $\mathrm{NC}$ with $\mathrm{BHD}$ induction, $\left.{ }^{*} P<0.05\right)$. All data are presented as mean $\pm \mathrm{SD}$.

A

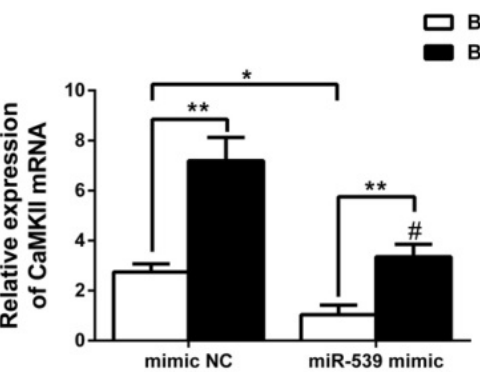

C

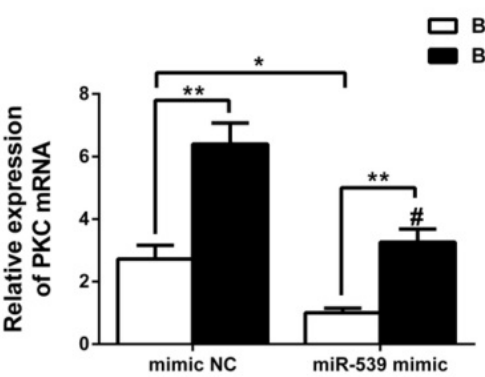

B

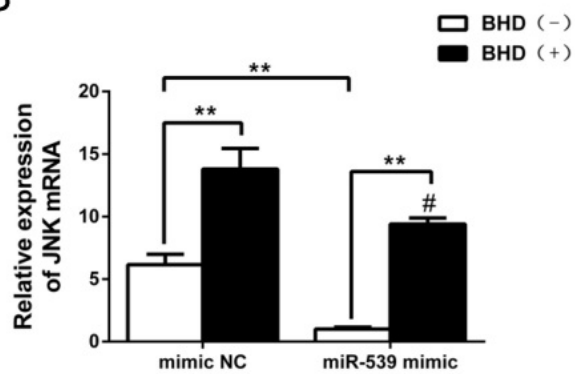

$\mathrm{D}$

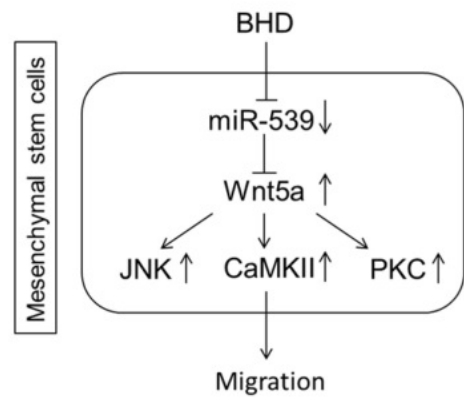

Figure 6. The down-stream molecules of Wnt5a were inhibited by miR-539-5p. (A-C) JNK, CaMKII and PKC mRNA was detected by real-time quantitative RT-PCR in rMSCs transfected with miR-539 mimics or NC in the presence of BHD or not. $\left(n=3, \# P<0.05\right.$ vs $r M S C s$ transfected with mimics $N C$ with $B H D$ induction, ${ }^{*}<0.05$, ** $\left.P<0.01\right)$. Data are presented as mean \pm SD. (D) Schematic diagram represents a model extrapolated from these results.

Next, we examined the levels of JNK, PKC and CaMKII mRNA in rMSCs transfected with miR-539-5p mimics. The results showed that overexpression of miR-539-5p decreased the expression of JNK, PKC and CaMKII in the presence or absence of BHD. Treatment with BHD partly restored the expression of JNK, PKC and CaMKII (Fig. 6A-C). 
Taken together, our results demonstrated that miR-539-5p took part in the regulation of migration in rMSCs treated with BHD through the regulation of Wnt5a and its downstream signaling molecules including JNK, PKC and CaMKII (Fig. 6D).

\section{Discussion}

This study investigated the mechanism involved in BHD regulation of rMSCs migration which is essential for bone healing [3]. Therefore, it is important to explore the positive and negative regulators of migration of rMSCs. In the previous study, we showed that BHD promotes migration of rMSCs by activating Wnt5a [13, 14]. In the present study, we further to identify miRNAs regulating migration of rMSCs induced by BHD. We discovered that miR-539-5p is down-regulated by BHD in rMSCs. Interestingly, we found that miR-539-5p was a negative regulator of migration of rMSCs. Further research showed that Wnt5a was a direct target of miR-539-5p. These findings suggest that miR-539-5p/Wnt5a signaling pathway is an important part of the regulatory machinery involved migration of rMSCs.

Previous research reported that miR-539-5p suppressed tumor cell migration and invasion $[28,29]$, promoted the development and progression of rheumatoid arthritis [30], as well as regulated osteoblast proliferation and differentiation and osteoclast apoptosis [31]. However, the role of miR-539-5p in the migration of rMSCs is still unknown. In this study, the decrease in the expression of miR-539-5p in BHD treated-rMSCs lead us to test whether miR-539-5p inhibits migration of rMSCs. We investigated the role of miR-539-5p in the process of migration. Transfection of miR-539-5p mimics inhibited migration of rMSCs. In contrast, transfection of miR-539-5p inhibitor promoted migration of rMSCs. Our data suggested miR-539-5p as a negative regulator of migration of rMSCs. These findings may provide a new regulatory role of miR-539-5p in the process of migration of rMSCs.

To further elucidate the intracellular molecular mechanism by which miR-539-5p regulates migration of rMSCs, we searched for potential target genes that have an established function in promoting migration of rMSCs using target gene prediction soft like Targetscan and Miranda. Interestingly, we discovered that the Wnt5a is one of the targets of miR-539-5p. Wnt5a takes part in the non-canonical Wnt pathway, including Wnt5a/Ca2 ${ }^{+}$signaling and Wnt5a/planar cell polarity $(\mathrm{PCP})$ signaling [32]. The $\mathrm{Wnt5a/Ca2}{ }^{+}$ signaling pathway involves activation of $\mathrm{Ca}^{+}$-dependent signaling molecules, including protein kinase $\mathrm{C}(\mathrm{PKC}), \mathrm{Ca}_{2}{ }^{+} /$calmodulin-dependent protein kinase II (CaMKII) [33]. Wnt5a/PCP signaling is mediated by activation of c-Jun N-terminal Kinases (JNKs) or RhoA signaling via small Rho-GTPases [34]. Wnt5a and its signaling pathway exerts migratory effects in large number of cell and tissue types in physiological and pathological contexts [22].Our previous study showed that BHD promotes migration of rMSCs by activating Wnt5a, so we selected Wnt5a as the target gene to further study [13, 14]. In this study, we showed that knock-down of Wnt5a resulted in a significant decrease in cell migration. The results are consistent with previous studies [13]. Then dual-luciferase reporter gene assay was conducted, which revealed that overexpression of miR-539-5p mimics suppressed the luciferase activity of the reporter construct. However, this effect was abolished when luciferase reporter containing a mutant 3'UTR of Wnt5a was co-transfected with miR-539-5p mimics, thus confirming the specificity of action. Further study showed that miR-539-5p also inhibited the expression of the downstream signaling molecules JNK, PKC and CaMKII of Wnt5a. In summary, this study provides evidence that BHD promotes migration of rMSCs through mir-539-5p/Wnt5a axis.

Furthermore, the findings of the present study have several important clinical implications. Firstly, fracture nonunions caused a significant economic burden and psychological burden to society, families and patients, for most patients have to rely on a wheelchair or bed-ridden life [35]. In recent years, stem cell-based therapy has gained much attention as the modern therapeutic approach to treat bone diseases [36]. Better understanding of the migration of MSCs and discovering conditions that improve their migration ability, will help to increase their homing to pathologies and improve Stem cell-based therapy and regenerative medicine outcomes [37]. Secondly, we find that miR-539-5p functions as a negative regulator of migration of rMSCs by suppressing Wnt5a expression. Therefore, pharmacological inhibition of miR-539-5p could represent a therapeutic strategy for improving rMSCs migration.

In conclusion, this study indicates that miR-539-5p functions as a negative regulator of migration of rMSCs by repressing Wnt5a expression, which in turn, results in suppression of the Wnt5a signaling pathway. Thus, miR-539-5p should be considered as an important candidate for the development of preventive or therapeutic approaches against bone nonunions.

\section{Supplementary Material}

Supplementary figure and table.

http://www.medsci.org/v16p0998s1.pdf 


\section{Acknowledgements}

This work was supported by the National Natural Science Foundation of China (81473699, 81473696 and 81503593) and the Natural Science Foundation of Guangdong Province (CN) (2014A020221055, 2016A030313649 and 2017A030313 729).

\section{Competing Interests}

The authors have declared that no competing interest exists.

\section{References}

1. Qu Z, Fang G, Cui Z, Liu Y. Cell therapy for bone nonunion: a retrospective study. Minerva Medica. 2015; 106: 315

2. Ding ZC, Lin YK, Gan YK, Tang TT. Molecular pathogenesis of fracture nonunion. J Orthop Translat. 2018; 14: 45-56.

3. Lin W, Xu L, Zwingenberger S, Gibon E, Goodman SB, Li G. Mesenchymal stem cells homing to improve bone healing. J Orthop Translat. 2017; 9: 19-27.

4. Fayaz HC, Giannoudis PV, Vrahas MS, Smith RM, Moran C, Pape HC, et al. The role of stem cells in fracture healing and nonunion. International Orthopaedics. 2011; 35: 1587

5. James AW. Review of Signaling Pathways Governing MSC Osteogenic and Adipogenic Differentiation. Scientifica (Cairo). 2013; 2013: 684736.

6. Lavrentieva A, Hatlapatka T, Neumann A, Weyand B, Kasper C. Potential for osteogenic and chondrogenic differentiation of MSC. Advances in Biochemical Engineering/biotechnology. 2013; 129: 73-88.

7. Shirley D, Marsh D, Jordan G, McQuaid S, Li G. Systemic recruitment of osteoblastic cells in fracture healing. J Orthop Res. 2005; 23: 1013-21.

8. Zhou Q, Yang C, Yang P. The Promotional Effect of Mesenchymal Stem Cell Homing on Bone Tissue Regeneration. Curr Stem Cell Res Ther. 2017; 12: 365-76.

9. Karp JM, Leng Teo GS. Mesenchymal stem cell homing: the devil is in the details. Cell Stem Cell. 2009; 4: 206-16.

10. Xu L, Huang S, Hou Y, Liu Y, Ni M, Meng F, et al. Sox11-modified mesenchymal stem cells (MSCs) accelerate bone fracture healing: Sox11 regulates differentiation and migration of MSCs. FASEB J. 2015; 29: 1143-52.

11. Huang S, Xu L, Sun Y, Zhang Y, Li G. The fate of systemically administrated allogeneic mesenchymal stem cells in mouse femoral fracture healing. Stem Cell Res Ther. 2015; 6: 206.

12. Granero-Molto F, Weis JA, Miga MI, Landis B, Myers TJ, O'Rear L, et al. Regenerative effects of transplanted mesenchymal stem cells in fracture healing. Stem Cells. 2009; 27: 1887-98.

13. Shen W, Luo H, Xu L, Wu Z, Chen H, Liu Y, et al. Wnt5a mediates the effects of Bushen Huoxue decoction on the migration of bone marrow mesenchymal stem cells in vitro. Chin Med. 2018; 13: 45.

14. Hui $\mathrm{L}$, Zhi-fang $\mathrm{W}$, Wei $\mathrm{S}$, Liu-chao $\mathrm{H}$, Bin $\mathrm{W}$, Li-juan $\mathrm{Y}$, et al. Effect of the petroleum ether extract from Bushen Huoxue Decoction on promoting BMSCs migration via Wnt5a / PKC signaling pathway. Chinese Traditional Patent Medicine. 2017: 2462-8.

15. Zhang R, Chen K, Lu D, Ma X, Hou L, Zhu X, et al. Study on Efficacy of "Bushen Huoxue Liquid" in Male Rats with Osteoporosis Induced by Dexamethasone and Its Mechanism. Journal of Chinese Medicinal Materials. 2003; $26: 347$.

16. Bing $X$, Hong-Ting J, Xiao-Feng W, Lu-Wei X, Pei-Jian T. Effects of serum of Bushen Huoxue prescription (Chinese characters) on classic Wnt/ $\beta$-catenin signaling pathways of osteoblasts. China Journal of Orthopaedics and Traumatology. 2015; 28: 553-8.

17. Oh K. Effect of Rehmannia glutinosa Libosch extracts on bone metabolism. Clinica Chimica Acta. 2003; 334: 185-95.

18. Yang HM, Shin H-K, Kang Y-H, Kim J-K. Cuscuta chinensis Extract Promotes Osteoblast Differentiation and Mineralization in Human Osteoblast-Like MG-63 Cells. Journal of Medicinal Food. 2009; 12: 85-92.

19. Li WD, Yan CP, Wu Y, Weng ZB, Yin FZ, Yang GM, et al. Osteoblasts proliferation and differentiation stimulating activities of the main components of Fructus Psoraleae corylifoliae. Phytomedicine. 2014; 21: 400-5.

20. Kikuchi A, Yamamoto H, Sato A, Matsumoto S. Wnt5a: its signalling, functions and implication in diseases. Acta Physiol (Oxf). 2012; 204: 17-33.

21. Matsumoto S, Fumoto K, Okamoto T, Kaibuchi K, Kikuchi A. Binding of APC and dishevelled mediates Wnt5a-regulated focal adhesion dynamics in migrating cells. EMBO J. 2010; 29: 1192-204.

22. Wei W, Li H, Li N, Sun H, Li Q, Shen X. WNT5A/JNK signaling regulates pancreatic cancer cells migration by Phosphorylating Paxillin. Pancreatology. 2013; 13: 384-92.

23. He L, Zhang H. MicroRNAs in the migration of mesenchymal stem cells. Stem Cell Reviews and Reports. 2019; 15: 3-12.
24. Meng F, Rui $Y, X u$ L, Wan $C$, Jiang X, Li G. Aqp1 enhances migration of bone marrow mesenchymal stem cells through regulation of FAK and beta-catenin. Stem Cells Dev. 2014; 23: 66-75.

25. Jui-Yu H, Tse-Shun H, Shu-Meng C, Wei-Shiang L, Tsung-Neng T, Lee OK, et al. miR-146a-5p circuitry uncouples cell proliferation and migration, but not differentiation, in human mesenchymal stem cells. Nucleic Acids Research. 2013; 41: 9753-63.

26. Mu-Han L, Chang-Jiang H, Ling C, Xi P, Jian C, Jiong-Yu H, et al. miR-27b Represses Migration of Mouse MSCs to Burned Margins and Prolongs Wound Repair through Silencing SDF-1a. Plos One. 2013; 8: e68972.

27. Zhu A, Kang N, He L, Li X, Xu X, Zhang H. MiR-221 and miR-26b Regulate Chemotactic Migration of MSCs Toward HGF Through Activation of Akt and FAK. Journal of Cellular Biochemistry. 2016; 117: 1370-83.

28. Yang ZX, Zhang B, Wei J, Jiang GQ, Wu YL, Leng BJ, et al. MiR-539 inhibits proliferation and migration of triple-negative breast cancer cells by down-regulating LAMA4 expression. Cancer Cell Int. 2018; $18: 16$.

29. Cui X, Zhang A, Liu J, Wu K, Chen Z, Wang Q. Down-regulation of MAP2K1 by miR-539 inhibits hepatocarcinoma progression. Biochemical and biophysical research communications. 2018; 504: 784-91.

30. Liu Y, Qian K, Li C, Ma Y, Chen X. Roles of microRNA-539 and osteopontin in rheumatoid arthritis. Exp Ther Med. 2018; 15: 2681-7.

31. Zhu XB, Lin WJ, Lv C, Wang L, Huang ZX, Yang SW, et al. MicroRNA-539 promotes osteoblast proliferation and differentiation and osteoclast apoptosis through the AXNA-dependent Wnt signaling pathway in osteoporotic rats. J Cell Biochem. 2018; 119: 8346-58.

32. Zhu N, Qin LI, Luo Z, Guo Q, Yang L, Liao D. Challenging role of Wnt5a and its signaling pathway in cancer metastasis (Review). Experimental and Therapeutic Medicine. 2014; 8: 3-8.

33. Kumawat K, Gosens R. WNT-5A: signaling and functions in health and disease. Cell Mol Life Sci. 2016; 73: 567-87.

34. McNeill $\mathrm{H}$, Woodgett JR. When pathways collide: collaboration and connivance among signalling proteins in development. Nat Rev Mol Cell Biol. 2010; 11: 404-13.

35. Wu N, Lee Y, Segina D, Murray H, Wilcox T, Boulanger L. Economic burden of illness among US patients experiencing fracture nonunion. Orthopedic Research and Reviews. 2013; 5: 21-33.

36. Yu SP, Wei Z, Wei L. Preconditioning strategy in stem cell transplantation therapy. Translational Stroke Research. 2013; 4: 76-88.

37. De Becker A, Riet IV. Homing and migration of mesenchymal stromal cells: How to improve the efficacy of cell therapy? World J Stem Cells. 2016; 8: 73-87. 